\title{
Clinical Characteristics of Non-Suicidal Self-Injury and Suicide Attempts among Psychiatric Patients in Korea: A Retrospective Chart Review
}

\author{
Yubeen Bae, Yoanna Seong, Seok Hyeon Kim, and Sojung Kim \\ Department of Psychiatry, Hanyang University Seoul Hospital, Seoul, Republic of Korea
}

Objective Limited data exist on non-suicidal self-injury (NSSI) and suicide attempts among psychiatric patients in Korea. In this study, we investigated the clinical characteristics of patients who engaged in NSSI and/or suicide attempts.

Methods We performed a retrospective medical chart review of patients with NSSI and/or suicide attempts at the psychiatric department of a university medical center in Seoul between 2017 and 2019. According to their history, patients were allocated to one of three groups: NSSI only, suicide attempts only and NSSI and suicide attempts group. Groups were compared based on sociodemographic characteristics and psychological assessments.

Results Overall, 80 patients with NSSI and/or suicide attempts were evaluated. Patients with NSSI and suicide attempts were more likely to be female than the other two groups. Patients with NSSI and suicide attempts were more likely to suffer from Cluster B personality disorder than the other groups. And patients with NSSI and suicide attempts scored significantly higher on novelty-seeking in TCI and RC8, RC9 in MMPI-2.

Conclusion Patients with NSSI and/or suicide attempts were more likely to be female, younger, and showed higher levels of psychological disturbances. These findings highlight the importance of early detection and intervention for patients with NSSI.

Psychiatry Investig 2020;17(4):320-330

Key Words Non-suicidal self-injury, Suicide attempts, Retrospective chart review.

\section{INTRODUCTION}

Non-suicidal self-injury (NSSI) has been defined as the deliberate, direct, socially unacceptable destruction or alteration of body tissue that occurs in the absence of suicidal intent. ${ }^{1}$ NSSI behaviors include cutting, burning, or hitting oneself, scratching wrists or arms and banging one's head against the wall. This behavior is a focus of a concern in adolescence given that onset frequently occurs between ages 12 and 14 , and the highest lifetime prevalence $(17.2 \%)$ is found during this period. ${ }^{2,3}$ NSSI is more commonly reported by females than males, and the gender difference is larger for

\footnotetext{
Received: October 9, 2019 Revised: February 3, 2020

Accepted: February 6, 2020

$\triangle$ Correspondence: Sojung Kim, PhD

Department of Psychiatry, Hanyang University Seoul Hospital, 222-1 Wangsimni-ro, Seungdong-gu, Seoul 04763, Republic of Korea

Tel: +82-2-2290-8429, Fax: +82-2-2290-8429, E-mail: sojungclair@gmail.com (ac) This is an Open Access article distributed under the terms of the Creative Commons Attribution Non-Commercial License (https://creativecommons.org/licenses/bync/4.0) which permits unrestricted non-commercial use, distribution, and reproduction in any medium, provided the original work is properly cited.
}

clinical samples compared to community samples. ${ }^{3}$ In addition, although NSSI increases in late adolescence and gradually declines in early adulthood, findings show that a group of people will continue to engage in NSSI into adulthood which leads to an increase in socioeconomic costs. ${ }^{4,5}$ NSSI is known to be associated with various psychological difficulties which are considered to be related to cognitive and psychological vulnerabilities including depression and anxiety, maladaptive coping strategies, self-criticism, impaired working memory, executive functioning and impulsiveness. ${ }^{6,7}$ It was reported that $20 \%$ of psychiatric patients engage in NSSI, while $5.5 \%$ of the community population experience the problem. ${ }^{3,8}$ Accumulated findings suggest that NSSI is associated with severe psychopathologies including depressive disorders, anxiety disorders, personality disorders, eating disorders and substance use disorders ${ }^{9-11}$ thereby highlighting the importance of research on NSSI among clinical populations.

According to a national survey by the South Korean Ministry of Education in 2018, 7.9\% of middle school students and $6.9 \%$ of high school students in Korea engaged in NSSI. ${ }^{12}$ Fur- 
ther, counseling cases of adolescents with NSSI tripled between 2017 and 2018 which reflects a sharp rise in the NSSI population in Korea. ${ }^{13}$ There has been growing interest among researchers in NSSI during the last decade, as social issues related to NSSI have begun to emerge in Korea, especially in adolescents. ${ }^{14}$ According to a recent systematic review of NSSI studies in Korea, which reviewed empirical studies conducted from 2000 to $2018,{ }^{14}$ depression and anxiety were reported as emotional factors predicting NSSI. ${ }^{15-19}$ Negative urgency, defined as a tendency to act rashly when experiencing negative emotionality, was also suggested as predicting NSSI. ${ }^{20}$ In addition, anger has been shown to significantly increase the frequency of the NSSI, which was more prevalent among women compared to men. ${ }^{19}$ Research on clinical patients has shown that traumatic experiences during childhood (for example, emotional abuse, physical neglect and sexual abuse) affect NSSI. Further, depression and obsession highly correlate with NSSI. ${ }^{21,22}$ In addition, studies of adolescents with depressive disorders demonstrate that anxiety and suicidal ideas are highly related to the NSSI. ${ }^{23}$ Despite the increasing number of people engaging in NSSI and the importance of research in the clinical population, according to Seonng et al., ${ }^{14}$ there have just been 23 empirical studies on NSSI, including only 4 studies conducted in clinical patients in Korea. Considering the limited number of studies on this topic, more research on NSSI in clinical patients are needed.

NSSI has been proposed as a preferred expression to characterize self-injurious behaviors that are performed without a suicidal intent, given the term's clarity and absence of pejorative meaning. ${ }^{2}$ In addition, NSSI and Suicidal Behavior Disorder (SBD) were included as separate conditions for further study in the 5th edition of the Diagnostic and Statistical Manual of Mental Disorders (DSM-5). ${ }^{24}$ However, controversy remains over the conceptual distinctions between NSSI and suicidal behavior. Some studies suggest that NSSI has different forms and functions in quality from suicide, and that there is a particular difference in intent and fatality. Gollust et al. ${ }^{25}$ reported self-harm is a different concept from suicide in that it is not at least an attempt to end one's life. In addition, according to Muehlenkamp and Kerr, ${ }^{26}$ NSSI and suicide attempts were viewed as distinct concepts in terms of intent, fatality, frequency, method, cognitive condition and outcome. Nock and Favazza ${ }^{1}$ also suggested that self-harm behavior differed from suicidal behavior in its prevalence in young people, low lethality, chronic pattern, sense of relief after the behavior, and different methods used by the same person.

Meanwhile, many other researchers argue that it is difficult to define NSSI and suicidal attempts as independent concepts. ${ }^{27}$ Allen $^{28}$ insisted that difficulties in differentiating NSSI from suicide attempts lie in the intention and fatality. He suggest- ed that, although the goal of suicide attempts is often to end one's pain with death, there are various reasons to end one's life (for example, to express anger, to punish someone). Also, in terms of fatalities, he argued that people can inadvertently lose their lives using potentially less lethal methods while they may survive when using more lethal methods with intent to die. ${ }^{29}$ This is consistent with empirical evidence that shows no significant relationship between the intent to die and success of suicide attempt in a large cohort study. ${ }^{30}$ Also, Kapur et al. ${ }^{27}$ argued that NSSI is not clearly distinctive from suicide attempts with the continuous distribution of suicide intent found in 771 patients who visited emergency room with selfharm. He suggested that, if the two concepts were dichotomized, the distribution should be bimodal, rather than be unimodal.

Recent evidence from empirical data suggests that NSSI is strongly related to suicide attempts. Research has found that NSSI is a powerful predictor of suicide attempts. ${ }^{31}$ When NSSI becomes chronic and evolves toward other forms of more serious self-injurious behaviors, it eventually leads to suicide attempts. $^{32,33}$ To investigate the relationship between NSSI and suicide attempts, patients with NSSI and patients with suicide attempts were compared. Results showed that patients with both NSSI and suicide attempts exhibit significantly more adverse psychological symptoms such as depression, hopelessness and suicidal ideations than patients with NSSI without suicide attempts. ${ }^{34}$ Also, patients with NSSI without suicide attempts were found to have fewer anhedonic symptoms, less suicidal ideation and less negative self-evaluations while they showed higher levels of self-esteem and parental support compared to patients with both NSSI and suicide attempts. ${ }^{35}$ Moreover, Andover and $\mathrm{Gibb}^{36}$ found that among patients with a history of suicide attempts, those who has engaged in NSSI reported significantly greater suicide intent. These results indicate that patients with NSSI might have less severe psychological symptoms than patients with both NSSI and suicide attempts. When comparing patients with NSSI and patients with suicide attempts, patients with suicide attempts were more likely to be diagnosed with major depressive disorders, show higher suicidal ideation, and have a history of suicide attempts or psychiatric care. ${ }^{37}$

There are a few studies on the relationship between NSSI and suicide attempts in Korea. Kim et al. ${ }^{38}$ found that repeated NSSI behaviors increase suicidal capability which ultimately raises the risk of suicide attempts. In addition, 57.4\% of adolescents with a history of self-harm reported suicidal ideas, ${ }^{16}$ while in an another study $32 \%$ of adolescents with a history of self-harm were found to have suicidal intent. ${ }^{17}$ The frequent observations of the coexistence of NSSI and suicidal behaviors require that we consider the nature of the link between 
these two types of behavior. To be specific, it seems necessary to investigate whether NSSI and suicidal behaviors are qualitatively distinguishable or are they within the same spectrum. However, there is a dearth of research into this area, especially, there are no empirical studies which have investigated this issue in the clinical population in Korea.

This is the first retrospective chart review in the clinical population of Korea with the aim of exploring differential characteristics of patient with NSSI and/or suicide attempts. To accomplish this, we compared patients with both NSSI and suicide attempts, patients with NSSI only, and patients with suicide attempts only based on sociodemographic characteristics, features of suicide attempts and NSSI, cognitive functioning, temperament and personality aspects.

\section{METHODS}

\section{Patients}

This study was conducted on early adult patients (18 to 39 years old) who have engaged in NSSI and/or SA and visited the department of psychiatry at one university medical center from 1 January 2017 to 31 May 2019. Participants were limited to patients who had completed psychological evaluations that contained the necessary information for this study. The study sample was composed of three different groups divided by their history of NSSI and/or SA through retrospective chart reviews: patients who have experienced 1) both NSSI and suicide attempts (NSSI+SA group), 2) only NSSI (NSSI-Only group), 3) only suicide attempts (SA-Only group). In addition, those diagnosed with psychotic disorder, autism spectrum disorder, intellectual disability, stereotypic movement disorder, trichotillomania and accompanying self-injurious behavior were excluded.

\section{Collection of study data}

A retrospective medical chart review was performed by two clinical psychologists using electronic medical records (EMR) and psychological evaluation reports, and by selecting patients who undertook suicide attempts or NSSI. The NSSI was defined as deliberate and intentional damage to one's body without suicidal intent, and suicide attempts were defined as suicidal behaviors with intent to die. Epidemiological data including gender, age, academic background, single marital status, inoccupation, hospitalization, present psychiatric disorders or psychiatric symptoms, treatment status, onset age of NSSI, methods of NSSI, onset age of suicide attempts, methods of suicide attempts, and the number of suicide attempts were documented. The diagnoses of psychiatric disorders were investigated by screening EMR records, previous medical conditions and psychological evaluation reports. Treatment dis- continuation was operationally defined as no visit to the department of psychiatry for more than two months since the last visitation date.

The study protocol was approved by the Institutional Review Board (IRB) of the authors' university medical center (IRB approval No. 2019-05-047-001) and conducted in accordance with the Declaration of Helsinki. Since this was a retrospective chart review, informed consent was waived by the IRB.

\section{Measures}

\section{Korean-Wechsler Adult Intelligence Scale-Fourth Edition (K-WAIS-IV)}

Wechsler Adult Intelligence Scale-Fourth Edition (WAISIV) was developed and validated in $2008 .^{39}$ The Korean version of the WAIS-IV (K-WAIS-IV) has good reliability and validity. ${ }^{40} \mathrm{~K}$-WAIS-IV is applicable to adults aged between 16 to 69 . With the Full Scale IQ (FSIQ) which represents general intelligence, we used scores of 10 core subtests, and four index scores: Verbal Comprehension (VC), Perceptual Reasoning (PR), Working Memory (WM), Processing Speed (PS). Each score of subtests is reported as a standard score with a mean of 10 and standard deviation of 3 . The four index scores and the FSIQ were combination scores with a mean of 100 and standard deviation of 15 .

\section{Temperament and Character Inventory (TCI)}

TCI was developed by Svrakic et al. ${ }^{41}$ for the purpose of explaining, diagnosing and predicting the development process of personality disorder. It consists of 240 questions for four temperament dimensions and three personality dimensions. The four temperament dimensions are Novelty Seeking (NS), Harm Avoidance (HA), Reward Dependence (RD) and Persistence $(\mathrm{P})$. Character dimensions include Self-Directedness (SD), Cooperativeness (C), and Self-Transcendence (ST). The internal consistencies of the 7 scales were ranged between 0.77 and 0.88 and the test-retest reliabilities among university students were good, which ranged between 0.76 and $0.90{ }^{42}$

\section{Minnesota Multiphasic Personality Inventory-2 (MMPI-2)}

MMPI was developed by Hathaway and McKinley ${ }^{43}$ and was conceptualized as an efficient way of detecting psychiatric disturbances. Later, it became a structured measure for assessing personality and psychopathological conditions. MMPI-2 was developed by Butcher et al. ${ }^{44}$ In the study, we used validity scales and clinical scales. Also, restructured clinical scales which were designed to assess the underlying distinctive core components of the MMPI-2 clinical scales were adopted in order to enhance discriminant and convergent validity. The test-retest reliabilities of 10 clinical scales were ranged 
between 0.54 and 0.93 and the internal consistencies (Cronbach's as) were ranged between 0.34 and $0.87 .{ }^{45}$

\section{Statistical analyses}

All analyses were performed using IBM SPSS Statistics version 25.0 (IBM Corp., Armonk, NY, USA), a p-value< 0.05 was considered as statistically significant. The Kolmogrov-Smirnov and Shapiro-Wilk tests were used to check the normality of our metric data. The data that were normally distributed with equal variances among groups were subjected to one-way ANOVAs followed by Fisher's LSD post hoc comparisons. The data that were neither normally distributed nor had equal variances were subjected to Kruskal-Wallis test followed by Games-Howell multiple-comparison post-test. Categorical variables were compared using $\chi^{2}$-test.

\section{RESULTS}

\section{Demographic characteristics}

Participants consisted of three groups: 27 patients with NSSI and suicide attempts, 14 patients with NSSI-only and 39 patients with suicide attempts-only. The participants' characteristics are shown in Table 1. There were significant differences in age and gender ratio between the groups. The NSSI and suicide attempts group showed a higher ratio of females and were younger than the other two groups. There were no significant differences between groups in academic background, marital status, inoccupation, hospitalization, treatment status. Regarding psychiatric disorders, the NSSI and suicide attempts group was more likely to be diagnosed with Cluster B personality disorders compared to the NSSI only group or the suicide attempts only group. The most common diagnosis was depressive disorder which was followed by Cluster B personality, anxiety disorder, and alcohol use disorder.

\section{Characteristics of NSSI behaviors}

To investigate the differences in NSSI behaviors between groups, patients with NSSI-only and patient with NSSI and suicide attempts were compared. Patients with NSSI and suicide attempts reported a younger age of onset compared to patients with NSSI at a trend level of significance, $\mathrm{p}=0.051$. Among the 41 participants with NSSI with or without sui-

Table 1. Demographic characteristics

\begin{tabular}{|c|c|c|c|c|c|c|}
\hline Variables & $\mathrm{NSSI}+\mathrm{SA}(\mathrm{N}=27)$ & NSSI $(\mathrm{N}=14)$ & $\mathrm{SA}(\mathrm{N}=39)$ & Total $(\mathrm{N}=80)$ & F or $\chi^{2}$ & $\mathrm{p}$ \\
\hline Age (M/SD) & $22.56(4.16)$ & $22.14(2.21)$ & $25.28(5.45)$ & $23.81(4.78)$ & 3.885 & 0.025 \\
\hline Female (N/\%) & $20(74.1)$ & $6(42.9)$ & $15(38.5)$ & $41(51.2)$ & 8.577 & 0.014 \\
\hline Education (N/\%) & & & & & 1.983 & 0.739 \\
\hline Middle school & $2(7.4)$ & $0(0)$ & $1(2.6)$ & $3(3.8)$ & & \\
\hline High school & $6(22.2)$ & $3(21.4)$ & $7(17.9)$ & $16(20)$ & & \\
\hline College $>$ college & $19(70.4)$ & $11(78.6)$ & $31(79.5)$ & $61(76.3)$ & & \\
\hline Inoccupation (N/\%) & $13(48.1)$ & $3(21.4)$ & $19(48.7)$ & $35(43.8)$ & 3.862 & 0.425 \\
\hline Never married (N/\%) & $26(96.3)$ & $13(92.9)$ & $34(87.2)$ & $73(91.3)$ & 1.716 & 0.424 \\
\hline Hospitalization (N/\%) & $17(63)$ & $7(50)$ & $21(53.8)$ & $45(56.3)$ & 0.808 & 0.668 \\
\hline Treatment status (N/\%)* & $15(55.6)$ & $10(71.4)$ & $19(48.7)$ & $44(55)$ & 2.152 & 0.341 \\
\hline \multicolumn{7}{|l|}{ Psychiatric disorders } \\
\hline Depressive disorder & $17(63)$ & $8(57.1)$ & $31(79.5)$ & $56(70)$ & 3.410 & 0.180 \\
\hline Bipolar disorder & $4(14.8)$ & $0(0)$ & $2(5.1)$ & $6(7.5)$ & 3.534 & 0.171 \\
\hline Anxiety disorder & $5(18.5)$ & $3(21.4)$ & $5(12.8)$ & $13(16.3)$ & 0.715 & 0.699 \\
\hline PTSD & $1(3.7)$ & $0(0)$ & $3(7.7)$ & $4(5)$ & 1.428 & 0.490 \\
\hline Adjustment disorder & $0(0)$ & $1(7.1)$ & $2(5.1)$ & $3(3.8)$ & 1.704 & 0.427 \\
\hline Eating disorder & $1(3.7)$ & $1(7.1)$ & $0(0)$ & $2(2.5)$ & 2.399 & 0.301 \\
\hline Alcohol use disorder & $3(11.1)$ & $0(0)$ & $4(10.3)$ & $7(8.8)$ & 1.642 & 0.440 \\
\hline Substance use disorder & $1(3.7)$ & $0(0)$ & $1(2.6)$ & $2(2.5)$ & 0.520 & 0.771 \\
\hline Impulse control disorder & $0(0)$ & $0(0)$ & $4(10.3)$ & $4(5)$ & 4.426 & 0.109 \\
\hline Cluster B personality disorder & $12(44.4)$ & $3(21.4)$ & $4(10.3)$ & $19(23.8)$ & 10.348 & 0.006 \\
\hline $\mathrm{ADHD}$ & $1(3.7)$ & $0(0)$ & $3(7.7)$ & $4(5)$ & 1.428 & 0.490 \\
\hline Other disorders & $1(3.7)$ & $0(0)$ & $3(7.7)$ & $4(5)$ & 1.428 & 0.490 \\
\hline
\end{tabular}

*treatment status-continuation. NSSI: non-suicidal self-injury, SA: suicide attempts, PTSD: post-traumatic stress disorder, ADHD: attention deficit hyperactivity disorder 
cide attempts, 34 reported specific NSSI methods. The most commonly endorsed method of NSSI was cutting (61\%) in patients with NSSI with or without suicide attempts, and there were no significant between-group differences in methods of NSSI. The total number of NSSI methods was also investigated. Twenty-two used a single method while the other 12 used two or more methods (Table 2).

\section{Characteristics of suicide attempts}

Patients with NSSI and suicide attempts and patients with suicide attempts-only were compared to explore the differences in characteristics of suicide attempts between groups. Patients with NSSI and suicide attempts reported younger ages of onset compared to patients with suicide attempts only, but the difference was not significant between groups, $\mathrm{p}=$ 0.126 . Patients with NSSI and suicide attempts reported more lifetime suicide attempts than patients with suicide attempts only. Regarding methods of suicide attempts, patients with NSSI and suicide attempts reported more drug intoxication and cutting than patients with suicide attempts only. Details are provided in Table 3.

\section{Cognitive functioning}

To explore the differences in cognitive functioning between groups, FSIQ and index scores of the K-WAIS-IV were compared between the three groups. There were no significant group differences in all scores. For FSIQ and four index scores (VCI, PRI, WMI, and PSI), normal levels range from 90 to 109. And for subtests, average scores range from 9 to $11 .^{40}$ The total sample of the current study showed lower-average levels of performance in the PSI, while the FSIQ and the other three index scores were at an average level (Table 4).

\section{Temperament and character}

To investigate the differences in temperament and character between groups, the T-scores of the TCI from all three

Table 2. Group differences in characteristics of NSSI behaviors

\begin{tabular}{|c|c|c|c|c|c|}
\hline Variables & $\mathrm{NSSI}+\mathrm{SA}(\mathrm{N}=27)$ & $\operatorname{NSSI}(\mathrm{N}=14)$ & Total $(\mathrm{N}=41)$ & F or $\chi^{2}$ & $\mathrm{p}$ \\
\hline Age of onset & $15.82(4.05)$ & $19(4.62)$ & $16.88(4.44)$ & 4.115 & 0.051 \\
\hline \multicolumn{6}{|l|}{ Methods of NSSI } \\
\hline Complex & $9(33.3)$ & $3(21.4)$ & $12(29.3)$ & 1.347 & 0.510 \\
\hline Cutting & $18(66.7)$ & $7(50)$ & $25(61)$ & 1.076 & 0.300 \\
\hline Scratching & $4(14.8)$ & $0(0)$ & $4(9.8)$ & 2.298 & 0.130 \\
\hline Hitting & $5(18.5)$ & $2(14.3)$ & $7(17.1)$ & 0.117 & 0.733 \\
\hline Drug & $3(11.1)$ & $2(14.3)$ & $5(12.2)$ & 0.087 & 0.768 \\
\hline Etc & $4(14.8)$ & $1(7.1)$ & $5(12.2)$ & 0.507 & 0.477 \\
\hline Not applicable & $3(11.1)$ & $4(28.6)$ & $7(17.1)$ & 1.985 & 0.159 \\
\hline $\begin{array}{l}\text { Total number of NSSI methods } \\
\text { (single:multiple) }\end{array}$ & $15: 9$ & $7: 3$ & $22: 12$ & 0.174 & 0.677 \\
\hline
\end{tabular}

NSSI: non-suicidal self-injury, SA: suicide attempts

Table 3. Group differences in characteristics of suicide attempts

\begin{tabular}{|c|c|c|c|c|c|}
\hline Variables & $\mathrm{NSSI}+\mathrm{SA}(\mathrm{N}=27)$ & $\mathrm{SA}(\mathrm{N}=39)$ & Total $(\mathrm{N}=66)$ & F or $\chi^{2}$ & $\mathrm{p}$ \\
\hline Age of onset & $20.91(2.76)$ & $23.39(7.28)$ & $22.38(5.95)$ & 2.417 & 0.126 \\
\hline Frequency & $2.24(1.26)$ & $1.59(1.34)$ & $1.85(1.33)$ & 2.551 & 0.011 \\
\hline Visiting emergency room & $23(85.2)$ & $28(71.8)$ & $51(77.3)$ & 2.732 & 0.255 \\
\hline \multicolumn{6}{|l|}{ Methods of suicidal attempt } \\
\hline Drug intoxication & $16(59.3)$ & $12(30.8)$ & $28(42.4)$ & 5.302 & 0.021 \\
\hline Hanging & $5(18.5)$ & $8(20.5)$ & $13(19.7)$ & 0.040 & 0.841 \\
\hline Jumping & $2(7.4)$ & $9(23.1)$ & $11(16.7)$ & 2.821 & 0.093 \\
\hline Gas & $6(22.2)$ & $12(30.8)$ & $18(27.3)$ & 0.588 & 0.443 \\
\hline Cutting & $7(25.9)$ & $2(5.1)$ & $9(13.6)$ & 5.860 & 0.015 \\
\hline Etc & $1(3.7)$ & $1(2.6)$ & $2(3)$ & 0.071 & 0.791 \\
\hline Not applicable & $3(11.1)$ & $6(15.4)$ & $9(13.6)$ & 0.247 & 0.619 \\
\hline
\end{tabular}

NSSI: non-suicidal self-injury, SA: suicide attempts 
groups were compared. Patients with NSSI and suicide attempts reported significantly higher scores on novelty-seeking than patients with suicide attempts only. However, there were no significant between-group differences in other indicators. All three groups showed high levels of HA and low levels of SD. Patients with NSSI and suicide attempts had high levels of NS, HA and low SD, while patients with NSSI only reported high levels of HA and low levels of SD. Patients in the suicide attempts only group reported high levels of HA and low levels of SD and C (Table 5).

\section{Personality and psychological symptoms}

The T-scores of the MMPI-2 were compared to explore the group differences in personality and psychological symptoms. There were no significant group differences in all va- lidity scales and clinical scales (Table 6). In order to explore group differences in detailed areas, the group differences on the restructured clinical scales were analyzed. Patients with NSSI and suicide attempts reported significantly higher scores on RC8 than patients with suicide attempts only. And patients with NSSI and suicide attempts reported significantly higher scores on RC9 than patients with NSSI only, and patients with suicide attempts only.

\section{DISCUSSION}

This is the first retrospective chart review to investigate the clinical characteristics of psychiatric patients with NSSI and suicide attempts in Korea. To explore the diagnostic correlates of NSSI and suicide attempts, patients with NSSI, patients

Table 4. Group differences in cognitive functioning on K-WAIS-IV

\begin{tabular}{ccccccc}
\hline Variables & NSSI+SA $(\mathrm{N}=27)$ & NSSI $(\mathrm{N}=14)$ & SA $(\mathrm{N}=39)$ & Total $(\mathrm{N}=80)$ & $\mathrm{F}$ or $\chi^{2}$ & $\mathrm{p}$ \\
\hline FSIQ & $93.85(14.75)$ & $93.57(15.22)$ & $93.72(14.93)$ & $93.74(14.73)$ & 0.002 & 0.998 \\
VCI & $96.67(12.89)$ & $100.36(14)$ & $97.62(13.19)$ & $97.78(13.13)$ & 0.364 & 0.696 \\
SI & $9.44(3.42)$ & $10.29(3.19)$ & $9.74(2.57)$ & $9.74(2.96)$ & 0.365 & 0.696 \\
VC & $9.00(3.28)$ & $10.00(3.21)$ & $9.05(2.94)$ & $9.20(3.09)$ & 0.564 & 0.571 \\
IN & $9.11(2.29)$ & $9.57(2.1)$ & $9.64(2.65)$ & $9.45(2.42)$ & 0.409 & 0.792 \\
PRI & $98.89(14.55)$ & $96.57(16.25)$ & $101.82(17.55)$ & $99.91(16.92)$ & 0.609 & 0.547 \\
BD & $9.44(2.94)$ & $9.07(3.33)$ & $9.87(3.56)$ & $9.59(3.29)$ & 0.336 & 0.716 \\
MR & $9.04(2.71)$ & $9.64(2.95)$ & $10.05(3.44)$ & $9.64(3.12)$ & 0.838 & 0.436 \\
VP & $10.52(2.79)$ & $9.29(2.7)$ & $10.59(2.9)$ & $10.34(2.84)$ & 1.173 & 0.315 \\
WMI & $97.52(16.75)$ & $95.14(14.83)$ & $94.77(14.36)$ & $95.76(15.14)$ & 0.272 & 0.763 \\
DS & $10.33(3.49)$ & $9.29(2.92)$ & $9.08(2.57)$ & $9.54(2.99)$ & 1.483 & 0.233 \\
AR & $8.52(3.08)$ & $8.79(3.76)$ & $8.82(3.32)$ & $8.71(3.28)$ & 0.430 & 0.806 \\
PSI & $90.63(17.3)$ & $87.5(15.24)$ & $86.67(19.98)$ & $88.15(18.22)$ & 0.467 & 0.792 \\
SS & $8.81(4.14)$ & $7.79(2.88)$ & $7.21(3.96)$ & $7.85(3.88)$ & $7.29(3.35)$ & 0.034 \\
CD & $7.15(2.9)$ & $7.36(3.29)$ & $7.36(3.71)$ & 0.256 & 0.966 \\
\hline
\end{tabular}

WAIS-IV: Wechsler Adult Intelligence Scale-Fourth Edition, NSSI: non-suicidal self-injury, SA: suicide attempt, FSIQ: full-scale intelligence quotient, VCI: verbal comprehension index, PRI: perceptual reasoning index, WMI: working memory index, PSI: processing speed index, VC: vocabulary, SI: similarities, IN: information, CO: comprehension, BD: block design, MR: matrix reasoning, VP: visual puzzles, AR: arithmetic, DS: digit span, SS: symbol span, CD: coding

Table 5. Group differences in temperament and character on $\mathrm{TCl}$

\begin{tabular}{|c|c|c|c|c|c|c|}
\hline Variables & $\mathrm{NSSI}+\mathrm{SA}(\mathrm{N}=27)$ & NSSI (N=14) & $\mathrm{SA}(\mathrm{N}=39)$ & Total $(\mathrm{N}=80)$ & F or $\chi^{2}$ & $\mathrm{p}$ \\
\hline NS & $66.78(14.53)$ & $60.29(14.38)$ & $57.49(12.95)$ & $61.11(14.2)$ & 3.672 & 0.030 \\
\hline HA & $71.44(13.41)$ & $74.21(16.71)$ & $68.54(14.5)$ & $70.51(14.52)$ & 0.867 & 0.424 \\
\hline $\mathrm{RD}$ & $44.15(14.8)$ & $40.79(16.11)$ & $36.77(13.73)$ & $39.96(14.93)$ & 2.025 & 0.139 \\
\hline $\mathrm{P}$ & $38.37(15.78)$ & $34.64(9.67)$ & $37.33(13.73)$ & $37.21(13.73)$ & 0.335 & 0.717 \\
\hline $\mathrm{SD}$ & $25.19(9.8)$ & $29.5(17.11)$ & $28.03(12.35)$ & $27.33(12.48)$ & 0.664 & 0.518 \\
\hline C & $38.78(17.69)$ & 35.07 (15.88) & $34.23(15.21)$ & $35.91(16.13)$ & 0.651 & 0.524 \\
\hline ST & $42.74(17.13)$ & $35.21(15.8)$ & $38.08(15.69)$ & $39.15(16.24)$ & 1.160 & 0.319 \\
\hline
\end{tabular}

TCI: Temperament and Character Inventory, NSSI: non-suicidal self-injury, SA: suicide attempt, NS: novelty seeking, HA: harm avoidance, RD: reward dependence, P: persistence, SD: self-directedness, C: cooperativeness, ST: self-transcendence 
Table 6. Group differences in personality and psychological symptoms on MMPI-2

\begin{tabular}{|c|c|c|c|c|c|c|}
\hline Variables & $\mathrm{NSSI}+\mathrm{SA}(\mathrm{N}=27)$ & NSSI $(\mathrm{N}=14)$ & $\mathrm{SA}(\mathrm{N}=39)$ & Total $(\mathrm{N}=80)$ & $\mathrm{F}$ or $\chi^{2}$ & $\mathrm{p}$ \\
\hline \multicolumn{7}{|l|}{ Validity scales } \\
\hline $\mathrm{F}$ & $64.52(11.6)$ & $63.64(12.79)$ & $61.67(14.92)$ & $62.98(13.42)$ & 2.008 & 0.366 \\
\hline $\mathrm{L}$ & $41.63(7.14)$ & $45.00(8.51)$ & $45.56(9.4)$ & $44.14(8.63)$ & 2.797 & 0.247 \\
\hline $\mathrm{K}$ & $39.48(6.36)$ & $41.64(9.65)$ & $43.38(7.63)$ & $41.76(7.73)$ & 4.247 & 0.120 \\
\hline$S$ & $38.04(8.32)$ & $38.86(12.08)$ & $42.64(9.54)$ & $40.43(9.77)$ & 4.799 & 0.091 \\
\hline \multicolumn{7}{|l|}{ Clinical scales } \\
\hline Hs & $60.74(9.65)$ & $64.36(10.55)$ & $58.23(13.85)$ & $60.15(12.09)$ & 2.484 & 0.289 \\
\hline $\mathrm{D}$ & $67.96(12.31)$ & $73.00(10.87)$ & $67.77(14.04)$ & $68.75(12.96)$ & 1.984 & 0.371 \\
\hline Hy & $58.41(7.02)$ & $59.86(8.97)$ & $58.00(12.07)$ & $58.46(10)$ & 0.174 & 0.840 \\
\hline PD & $61.93(10.92)$ & $65.07(11.33)$ & $66.82(12.38)$ & $64.86(11.79)$ & 1.391 & 0.255 \\
\hline Mf & 48.78 (12.79) & $51.86(10.5)$ & $51.92(11.49)$ & $50.85(11.74)$ & 0.629 & 0.536 \\
\hline $\mathrm{Pa}$ & $69.70(11.42)$ & $69.86(13.61)$ & $64.49(14.52)$ & $67.19(13.48)$ & 1.547 & 0.219 \\
\hline $\mathrm{Pt}$ & $71.15(11.69)$ & $74.79(14.84)$ & $66.72(15.79)$ & $69.63(14.52)$ & 3.346 & 0.188 \\
\hline Sc & $70.07(13.73)$ & $70.00(13.26)$ & $65.56(14.92)$ & $67.86(14.25)$ & 0.989 & 0.377 \\
\hline $\mathrm{Ma}$ & $55.85(10.23)$ & $50.93(10.67)$ & $50.21(9.17)$ & $52.24(10.02)$ & 5.090 & 0.078 \\
\hline $\mathrm{Si}$ & $63.30(12)$ & $70.79(17.59)$ & $64.79(16.31)$ & $65.34(15.27)$ & 1.161 & 0.319 \\
\hline \multicolumn{7}{|c|}{ Restructured clinical scales } \\
\hline RCd & $76.59(8.85)$ & $75.43(11.69)$ & $71.67(13.29)$ & $73.99(11.77)$ & 1.543 & 0.220 \\
\hline $\mathrm{RC} 1$ & $67.56(13.98)$ & $69.29(14.67)$ & $60.97(17.52)$ & $64.65(16.15)$ & 2.079 & 0.132 \\
\hline RC2 & $66.15(12.56)$ & $70.43(13.78)$ & $67.21(15.46)$ & $67.41(14.15)$ & 0.423 & 0.656 \\
\hline RC3 & $59.22(14.9)$ & $63.5(15.36)$ & $55.79(13.12)$ & $58.30(14.24)$ & 1.616 & 0.205 \\
\hline $\mathrm{RC4}$ & $61.63(14.79)$ & $62.14(14.38)$ & $61.67(14.37)$ & $61.74(14.33)$ & 0.007 & 0.993 \\
\hline RC6 & $61.44(13.65)$ & $57.36(18.85)$ & $55.44(14.78)$ & $57.80(15.24)$ & 1.255 & 0.291 \\
\hline RC7 & $69.33(15.01)$ & $69.07(15.22)$ & $64.08(15.73)$ & $66.73(15.43)$ & 1.125 & 0.330 \\
\hline $\mathrm{RC} 8$ & $68.15(16.06)$ & $60.07(14.64)$ & $58.23(15.51)$ & $61.90(16.01)$ & 6.690 & 0.035 \\
\hline RC9 & $58.37(12.94)$ & $51.21(10.57)$ & $48.69(8.66)$ & $52.40(11.36)$ & 8.428 & 0.015 \\
\hline
\end{tabular}

MMPI-2: Minnesota Multiphasic Personality Inventory-2, NSSI: non-suicidal self-injury, SA: suicide attempt, L: lie scale, F: infrequency scale, K: correction scale, S: superlative self-presentation scale, Hs: hypochondriasis, D: depression, Hy: hysteria, Pd: psychopathic deviate, Pa: paranoia, Pt: psychasthenia, Sc: schizophrenia, Ma: mania, Si: social introversion, RCd: demoralization, RC1: somatic complaints, RC2: low positive emotions, RC3: cynicism, RC4: antisocial behavior, RC6: ideas of persecution, RC7: dysfunctional negative emotions, RC8: aberrant experiences, RC9: hypomanic activation

with suicide attempts and patients with NSSI and suicide attempts were compared using sociodemographic characteristics, features of suicide attempts and NSSI, cognitive functioning, temperament and personality aspects. Several important findings warrant brief comment.

Patients with NSSI and suicide attempts and patients with NSSI only were more likely to be female than patients with suicide attempts only. This is in line with the findings that females show a higher rate of prevalence of NSSI and that gender differences are more prominent among clinical patients. ${ }^{46}$ Ye et al. ${ }^{23}$ who investigated psychosocial factors of NSSI among clinical patients in Korea also reported higher prevalence of females. Sornberger et al. ${ }^{47}$ reported that female adolescents prefer NSSI methods that result in bleeding such as cutting or scratching, while male adolescents more frequently use burning or hitting. The motivations for NSSI were also different between genders in psychiatric patients; females perform NSSI with intra-personal motivations, such as to punish oneself or to regulate negative emotions, whereas males do it with interpersonal motivations, such as to impact other people or to communicate. ${ }^{48}$ Due to the small number of each group, gender differences were not analyzed in detail in the study. Future research could explore gender differences in the methods of NSSI and it use as an emotion regulation strategy. We also found that patients with NSSI and patients with both NSSI and suicide attempts were significantly younger than patients with suicide attempts only. This finding was consistent with results from previous research that NSSI increases until late adoles- 
cence and then gradually decreases. The prevalence of suicide attempt is higher than that of NSSI in early adulthood. ${ }^{5,49}$ Also, in our study, patients with NSSI and suicide attempt reported younger age of onset compared to that of patients with NSSI only. It is known that the earlier onset of NSSI is related to a worse prognosis with more serious NSSI and suicidal plans. ${ }^{50}$ These data indicate that it is necessary to provide early interventions for young adolescents who start to develop selfharming behaviors. However, cautious interpretations should be made as individuals with NSSI who committed acts that were not potentially as lethal as suicide attempts might not have received medical attention. Future research should investigate NSSI and/or suicide attempts at early ages in a large community sample to provide additional evidence.

Among our participants, $44 \%$ of patients with NSSI and suicide attempts and $21 \%$ of patients with NSSI were diagnosed with borderline personality disorder (BPD). This association between NSSI and BPD has been consistently demonstrated in existing research. Our findings are a little lower but still similar to those of previous research that showed that $51.7 \%$ of adolescents with NSSI were diagnosed with BPD and $54 \%$ of patients with BPD experienced NSSI., ${ }^{90}$ The diagnostic criteria of BPD in the DSM-5 includes impulsivity with a sense of urgency, self-harming behavior under emotional distress, and engagement in dangerous, risky, and potentially self-damaging activities. ${ }^{24}$ It suggests that NSSI and BPD share many aspects in terms of disinhibition. Turner et al. ${ }^{11}$ compared patients with BPD showing NSSI behaviors and patients without BPD but engage in NSSI behaviors. Patients with BPD and NSSI behaviors reported higher levels of depression, suicidal ideations and difficulties in emotion regulations. This implies that NSSI and BPD interplay with each other and aggravate the impairment of patients. Therefore, in clinical settings, close examinations and follow-ups should be provided to patients with BPD or NSSI.

Our data showed that the most commonly endorsed method of NSSI was cutting in patients with NSSI with or without suicide attempts in line with previous studies. ${ }^{14,47}$ However, we found no significant group difference in the total number of NSSI methods, while previous studies which have shown that the number of lifetime suicide attempts was associated with the number of NSSI methods. ${ }^{51,52}$ Regarding suicide attempts, we found that patients with NSSI and suicide attempts reported more frequent suicide attempts and used less lethal methods such as drug intake and cutting than patients with suicide attempts only. Andover and $\mathrm{Gibb}^{36}$ found that NSSI was related to more frequent suicide attempts and depressive symptoms while hopelessness was also related to suicide attempts among psychiatric inpatients. Furthermore, as repeated suicide attempts increase the capability for suicide through gradual habituation, early detection and interventions of NSSI seems crucial. ${ }^{53,54}$ Patients in the suicide attempts only group showed less frequent suicide attempts but more lethal methods of suicide attempts such as jumping or hanging. Therefore, it seems necessary to investigate lethality of methods and motivations for suicide attempts using structured measures such as Risk-Rescue Rating Scale and Suicide Attempt and Self-Injury Interview among individuals with NSSI and suicide attempts. $^{55}$

There were no significant group differences in cognitive functioning in K-WAIS-IV. This is consistent with the findings from Dougherty et al. ${ }^{56}$ that there were no significant group differences on WAIS among individuals with NSSI only and individuals with NSSI and suicide attempts. However, there are a number of studies that indicate NSSI was related to impaired executive functioning and impulsivity. ${ }^{7}$ Considering that significant results were found in specified tasks on executive functioning (The Stop-Signal Task, Go-Stop Impulsivity Paradigm), non-significant findings of the study might have been found due to the use of less sensitive tasks on K-WAISIV. Yet, we found decreased performance in processing speed, compared to the norm, in the total sample of the current study. This might be related to the high rate, 70\%, of depression comorbidity in our sample. But this finding might also reflect the decreased efficiency in cognitive functioning among patients with NSSI and/or suicide attempts. It is recommended to use more targeted and sensitive tasks for cognitive functioning and to compare with individuals without problems related to selfinjurious behaviors in future investigation.

With regard to temperament, patients with NSSI and suicide attempts reported significantly higher scores on noveltyseeking than patients with suicide attempts only. This suggests that the preferences of sensation-seeking and impulsive tendencies are associated with NSSI. Considering the existing results that individuals with NSSI experience positive emotions after undertaking NSSI behaviors, temperament related to novelty-seeking might have enhanced motivations for NSSI. These results could be considered together with our findings that individuals with NSSI and suicide attempts scored higher on RC9 in MMPI-2. In addition, $11 \%$ of them were diagnosed with alcohol use disorders. With increased energy levels and difficulties in impulse control, patients with NSSI seem to show maladaptive acting-out behaviors for instant gratification. As mentioned earlier, their difficulties in inhibition are related to impaired executive functioning. However, regarding the fact that more patients in NSSI and suicide attempts group were diagnosed with bipolar disorder than the other two groups, it is necessary to consider the possibility that the clinical characteristics of bipolar disorder might have affected RC9 scores. In terms of personality and clinical 
features, patients with NSSI and suicide attempts reported significantly higher scores on RC8 which reflects psychological disorganization and general distress. Together with the results from reviews on NSSI, increased psychological distress in daily life is highly associated with NSSI and suicidal behaviors. Especially, individuals with NSSI who lack adaptive coping strategies engage in NSSI for the purpose of eradicating negative emotions. ${ }^{20}$

In investigating the clinical characteristics of patients who engaged in NSSI and/or suicide attempts, this chart review addresses a prevalent and pressing problem with serious societal implications. The focus on psychiatric patients and their characteristics extrapolated from sociodemographic variables, NSSI and suicidal behaviors, and results from widely used structured psychological measures, enhances clinical implications. Particularly, patients were divided into three groups according to their experience with NSSI and suicide attempts, which allowed us to speculate about the common factors and distinguishable factors in NSSI and suicide attempts.

This study highlights the importance of early intervention for patients who are female, younger age of onset of NSSI and patients with both NSSI and suicide attempts. It also raises the challenge of systematic assessment for cognitive functioning and emotional states. Patients with NSSI showed high impulsivity, difficulties in behavioral control, psychological disorganization with increased energy. These results could interact and increase the risk of acting-out in stressful situations. In addition, interventions for promoting adaptive emotion regulation are especially needed with consideration of the lack of adaptive emotion regulation strategies among individuals with NSSI and suicide attempts. Also, the total sample of our study showed lower-average levels of performance in the processing speed index of K-WAIS-IV. And this might reflect the difficulties in effective coping in everyday life among individuals with NSSI and/or suicide attempts. ${ }^{57}$ Behavioral activation and cognitive skill training for enhancing attention might be beneficial for their daily functioning.

The limitations of the chart review lie mainly in its retrospective nature. There were a few participants whose data on NSSI or suicide attempts were missing. Furthermore, some variables, known as important predictors of NSSI, such as family history, antecedents, motivations and consequences of previous NSSI and suicide attempts and the number of suicide attempts after psychological assessment could not be collated. In addition, there were difficulties in including information on successful suicide attempts, and this might have influenced the group difference in the lifetime frequency of suicide attempts in our study. This impeded our ability to examine the specific impact of each variable and integrate the process of NSSI and suicide attempts. In addition, this chart review investigated consistent follow-ups of participants in psychiatric departments, but not the current medications or their impact on psychological symptoms.

Results from this review could lead to the development of a standardized assessment tool including structured clinical interviews, specific cognitive tasks devised for patients with NSSI and suicidal behaviors. Primary prevention and intensive intervention programs should address the high risk factors highlighted above (i.e., younger age of onset, female, NSSI with suicide attempts, decreased processing speed, noveltyseeking, psychological disorganization and distress). To fully understand the development of NSSI and suicide attempts, especially in the sociocultural context of Korea, future research could benefit from examining the impact of social media, academic or occupational states and peer relationships.

\section{Acknowledgments}

The authors thank Ms. Eunyoung Son, Hanyang University Seoul Hospital, for her assistance in data collection.

This work was supported by the Ministry of Education of the Republic of Korea and the National Research Foundation of Korea (NRF-2019S1A5 A8035638).

\section{Conflicts of Interest}

The authors have no potential conflicts of interest to disclose.

\section{Author Contributions}

Conceptualization: Yubeen Bae, Yoanna Seong, Sojung Kim. Methodology: Yubeen Bae, Sojung Kim. Formal analysis: Yubeen Bae, Sojung Kim. Data curation: all authors. Writing_original draft: Yubeen Bae, Yoanna Seong. Writing_-review and editing: Sojung Kim, Seok Hyeon Kim. Approval of final manuscript: all authors.

\section{ORCID iDs}

Yubeen Bae

Yoanna Seong

Seok Hyeon Kim

Sojung Kim

https://orcid.org/0000-0001-5056-1314 https://orcid.org/0000-0003-3548-4052

https://orcid.org/0000-0002-0530-8026

https://orcid.org/0000-0002-5747-1052

\section{REFERENCES}

1. Nock MK, Favazza AR. Nonsuicidal Self-Injury: Definition and Classification. Washington, DC: American Psychological Association; 2009.

2. Jacobson CM, Gould M. The epidemiology and phenomenology of non-suicidal self-injurious behavior among adolescents: a critical review of the literature. Arch Suicide Res 2007;11:129-147.

3. Swannell SV, Martin GE, Page A, Hasking P, St John NJ. Prevalence of nonsuicidal self-injury in nonclinical samples: Systematic review, metaanalysis and meta-regression. Suicide Life Threat Behav 2014;44:273303.

4. Bragazzi NL. A Google Trends-based approach for monitoring NSSI. Psychol Res Behav Manag 2013;7:1-8.

5. Whitlock J, Eckenrode J, Silverman D. Self-injurious behaviors in a college population. Pediatrics 2006;117:1939-1948.

6. Valencia-Agudo F, Burcher GC, Ezpeleta L, Kramer T. Nonsuicidal self-injury in community adolescents: a systematic review of prospective predictors, mediators and moderators. J Adolesc 2018;65:25-38.

7. Fikke L, Melinder A, Landrø N. Executive functions are impaired in adolescents engaging in non-suicidal self-injury. Psychol Med 2011;41: 601-610. 
8. Klonsky ED, Muehlenkamp JJ. Self-injury: a research review for the practitioner. J Clin Psychol 2007;63:1045-1056.

9. Selby EA, Bender TW, Gordon KH, Nock MK, Joiner Jr TE. Non-suicidal self-injury (NSSI) disorder: a preliminary study. Personal Disord 2012;3:167-175.

10. Victor SE, Muehlenkamp JJ, Hayes NA, Lengel GJ, Styer DM, Washburn JJ. Characterizing gender differences in nonsuicidal self-injury: Evidence from a large clinical sample of adolescents and adults. Compr Psychiatry 2018;82:53-60.

11. Turner BJ, Dixon-Gordon KL, Austin SB, Rodriguez MA, Rosenthal MZ, Chapman AL. Non-suicidal self-injury with and without borderline personality disorder: differences in self-injury and diagnostic comorbidity. Psychiatry Res 2015;230:28-35.

12. Lee JH, Cho YY, Jeon JY. 70,000 middle and high school students "selfinflicted experiences". Available at: http://www.hani.co.kr/arti/society/ society_general/869668.html. Accessed November 10, 2018.

13. Park DH. The number of teenagers who were consulted for self-inflicted increased threefold. Available at: http://www.hani.co.kr/arti/society/rights/895831.html. Accessed May 29, 2019.

14. Seonng Y, Bae YB, Kim SJ. Non-suicidal self-injury in South Korea: a systematic review of studies from 2000 to 2018. Cogn Behav Ther Kor 2019;19:251-280.

15. Kim M, Yu J. Factors contributing to non-suicidal self injury in Korean adolescents. J Korean Acad Commun Health Nurs 2017;28:271-279.

16. Tak CH, Yoon BH, Kim KM, SEA YH, Jung HR, Song JH, et al. Predictive factors of self-injurious behavior with suicidal ideation in adolescents. Mood Emot 2017;15:149-155.

17. Lee DG, Ham KA, Bae BH. Adolescents self-injurious behaviors : suicidal self-injury and non-suicidal self-injury in female middle school students. Korean J Counsel Psychother 2016;28:1171-1192.

18. Koo HJ, WOO SB, Lee JS. The mediation effects of maladaptive cognitive emotion regulation and moderating effects of adaptive cognitive emotion regulation in the link between traumatic life events and selfharm: focused on gender difference. Korean J Clin Psychol 2015;34:173198.

19. Ahn YS, Somg HJ. Non-suicidal self-injury in adolescents. J Emot Behav Disord 2017;33:257-281.

20. Lim SY, Lee YH. The effects of negative urgency, negative affect, and emotion regulation difficulties on self- harm behavior in borderline personality disorder. Korean J Health Psychol 2007;22:565-585.

21. Kong SS. Impact of eating psychopathology, obsessive-compulsion and depression on self-harm behavior in patients with eating disorders. J Korean Acad Nurs 2009;39:459-468.

22. Kong SS, Lee JH, Shin MY. Impact of childhood trauma in self-harm behavior in patients with eating disorders and the mediating effect of parental conflict. J Korean Acad Psychiatr Ment Health Nurs 2009;18: 31-40.

23. Ye DH, Hong HY, Yook KH, Choi HI, Lee JE, Jun JY, et al. Psychosocial factors that influence on non-suicidal self-injury in depressed adolescents. J Korean Soc Depress Bipolar Disord 2012;10:85-90.

24. American Phychiatric Association. Diagnostic and Statistical Manual of Mental Disorders (DSM- $\left.{ }^{\circledR}\right)$. Washington, DC: American Psychiatric Pub; 2013.

25. Gollust SE, Eisenberg D, Golberstein E. Prevalence and correlates of self-injury among university students. J Am College Health 2008;56: 491-498.

26. Muehlenkamp JJ, Kerr PL. Untangling a complex web: how non-suicidal self-injury and suicide attempts differ. Prevent Res 2010;17:8-11.

27. Kapur N, Cooper J, O'connor RC, Hawton K. Non-suicidal self-injury v. attempted suicide: new diagnosis or false dichotomy? $\mathrm{Br} J$ Psychiatry 2013;202:326-328

28. Allen JG. Traumatic Relationships and Serious Mental Disorders. New Jersey: Wiley; 2001.

29. SJ K. A Review of the risk and protection factors of non-suicidal selfinjury. Korean J Youth Stud 2017;24:31-53.
30. Cooper J, Kapur N, Webb R, Lawlor M, Guthrie E, Mackway-Jones K, et al. Suicide after deliberate self-harm: a 4-year cohort study. Am J Psychiatry 2005;162:297-303.

31. Asarnow JR, Porta G, Spirito A, Emslie G, Clarke G, Wagner KD, et al. Suicide attempts and nonsuicidal self-injury in the treatment of resistant depression in adolescents: findings from the TORDIA study. J Am Acad Child Adolesc Psychiatry 2011;50:772-781.

32. Ribeiro J, Franklin J, Fox KR, Bentley K, Kleiman EM, Chang B, et al. Self-injurious thoughts and behaviors as risk factors for future suicide ideation, attempts, and death: a meta-analysis of longitudinal studies. Psychol Med 2016;46:225-236.

33. Whitlock J, Muehlenkamp J, Eckenrode J, Purington A, Abrams GB, Barreira P, et al. Nonsuicidal self-injury as a gateway to suicide in young adults. J Adolesc Health 2013;52:486-492.

34. Claes L, Muehlenkamp J, Vandereycken W, Hamelinck L, Martens H, Claes S. Comparison of non-suicidal self-injurious behavior and suicide attempts in patients admitted to a psychiatric crisis unit. Pers Individ Diff 2010;48:83-87.

35. Brausch AM, Gutierrez PM. Differences in non-suicidal self-injury and suicide attempts in adolescents. J Youth Adolesc 2010;39:233-242.

36. Andover MS, Gibb BE. Non-suicidal self-injury, attempted suicide, and suicidal intent among psychiatric inpatients. Psychiatry Res 2010; 178:101-105.

37. Chartrand H, Bhaskaran J, Sareen J, Katz LY, Bolton JM. Correlates of nonsuicidal self-injury and suicide attempts among tertiary care, emergency department patients. Can J Psychiatry 2015;60:276-283.

38. Kim CL, Lee SH, Chang H. Influence of non-suicidal self-injury on suicide attempt among depressed patients. Korean J Clin Psychol 2018;37: 465-478.

39. Wechsler D. Wechsler Adult Intelligence Scale-Fourth Edition (WAISIV). San Antonio, TX: NCS Pearson; 2008.

40. Hwang ST, Kim JH, Park GB, Choi JY, Hong SH. Korean Wechsler Adult Intelligence Scale-4th Edition (K-WAIS-IV). Daegu: Korea Psychology; 2012.

41. Svrakic DM, Whitehead C, Przybeck TR, Cloninger CR. Differential diagnosis of personality disorders by the seven-factor model of temperament and character. Arch Gen Psychiatry 1993;50:991-999.

42. Min BB, Oh HS, Lee JY. Temperament and Character Inventory-Revised-Short. Seoul: Maumsarang; 2007.

43. Hathaway S, McKinley J. Minnesota Multiphasic Inventory (MMPI) manual. Minneapolis: University of Minnesota Press; 1943.

44. Butcher J, Dahlstrom W, Graham J, Tellegen A, Kaemmer B. Manual for the Administration and Scoring of the MMPI-2. Minneapolis: University of Minnesota Press; 1989

45. Han KH, Lim JY, Min BB, Lee JH, Moon KJ, Kim ZS. Korean MMPI-2 Standardization Study. Korean J Clin Psychol 2006;25:533-564.

46. Bresin K, Schoenleber M. Gender differences in the prevalence of nonsuicidal self-injury: a meta-analysis. Clin Psychol Rev 2015;38:55-64.

47. Sornberger MJ, Heath NL, Toste JR, McLouth R. Nonsuicidal self-injury and gender: Patterns of prevalence, methods, and locations among adolescents. Suicide Life Threat Behav 2012;42:266-278.

48. Claes L, Vandereycken W, Vertommen H. Self-injury in female versus male psychiatric patients: a comparison of characteristics, psychopathology and aggression regulation. Pers Individ Diff 2007;42:611-621.

49. O'Connor RC, Wetherall K, Cleare S, Eschle S, Drummond J, Ferguson E, et al. Suicide attempts and non-suicidal self-harm: national prevalence study of young adults. BJ Psych Open 2018;4:142-148.

50. Ammerman BA, Jacobucci R, Kleiman EM, Uyeji LL, McCloskey MS. The relationship between nonsuicidal self-injury age of onset and severity of self-harm. Suicide Life Threat Behav 2018;48:31-37.

51. Hamza CA, Willoughby T. Nonsuicidal self-injury and suicidal behavior: a latent class analysis among young adults. PLoS One 2013;8:e59955.

52. Nock MK, Joiner TE Jr, Gordon KH, Lloyd-Richardson E, Prinstein MJ. Non-suicidal self-injury among adolescents: Diagnostic correlates and relation to suicide attempts. Psychiatry Res 2006;144:65-72. 
53. Joiner T. Why People Die by Suicide. Cambridge, MA: Harvard University Press; 2007.

54. Kim JY, Hong JP, Hwang JH, Jhoo JH, Kyeon YG, Lee KU. Retrospective recall study about psychological and behavioral characteristics in high lethality suicide attempters. J Korean Neuropsychiatr Assoc 2015; 54:435-443.

55. Weisman $\mathrm{AD}$, Worden JW. Risk-rescue rating in suicide assessment.
Arch Gen Psychiatry 1972;26:553-560.

56. Dougherty DM, Mathias CW, Marsh-Richard DM, Prevette KN, Dawes MA, Hatzis ES, et al. Impulsivity and clinical symptoms among adolescents with non-suicidal self-injury with or without attempted suicide. Psychiatry Res 2009;169:22-27.

57. Allen KJ, Bozzay ML, Edenbaum ER. Neurocognition and suicide risk in adults. Curr Behav Neurosci Rep 2019;6:151-165. 